In the version of this article initially published, the colors of the lines were switched in the graph that shows the glucose infusion rates for wildtype mice and Adipoq-Tst transgenic mice in Figure 3b. The top line should be purple, and the bottom line should be black. The error has been corrected in the HTML and PDF versions of the article.

\title{
Corrigendum: Colonic organoids derived from human induced pluripotent stem cells for modeling colorectal cancer and drug testing
}

Miguel Crespo, Eduardo Vilar, Su-Yi Tsai, Kyle Chang, Sadaf Amin, Tara Srinivasan, Tuo Zhang, Nina H Pipalia, Huanhuan Joyce Chen, Mavee Witherspoon, Miriam Gordillo, Jenny Zhaoying Xiang, Frederick R Maxfield, Steven Lipkin, Todd Evans \& Shuibing Chen Nat. Med. 23, 878-884 (2017); published online 19 June 2017; corrected after print 13 March 2018

In the version of this article initially published, there were several instances of duplication of confocal microscopy images presented in Figures 1e, $3 \mathrm{i}$ and $4 \mathrm{c}, \mathrm{h}, \mathrm{j}$. These errors occurred during the final preparation of the figures, when representative images were selected to illustrate the results obtained from multiple independent experiments. To ensure that these errors did not affect the paper's conclusions, the authors verified all raw data used to quantify the results. The results presented in the original Figures $3 \mathrm{j}$ and $4 \mathrm{~d}$ were not affected, but the quantification in Figure $4 \mathrm{i}$ was. This quantification was performed again, and the results supported the original conclusions of the paper. In addition, the western blot shown in Figure $3 \mathrm{~d}$ came from a different replicate experiment than the one represented in Supplementary Figure $9 \mathrm{~g}$, h (which shows the raw data). The original blot has been replaced with the correct blot that matches the raw data originally provided. The conclusions of this experiment remain unchanged. All affected images have been now corrected in the HTML and PDF versions of the article.

\section{Erratum: The molecular landscape of pediatric acute myeloid leukemia reveals recurrent structural alterations and age-specific mutational interactions}

\author{
Hamid Bolouri, Jason E Farrar, Timothy Triche Jr, Rhonda E Ries, Emilia L Lim, Todd A Alonzo, Yussanne Ma, Richard Moore, \\ Andrew J Mungall, Marco A Marra, Jinghui Zhang, Xiaotu Ma, Yu Liu, Yanling Liu, Jaime M Guidry Auvil, Tanja M Davidsen, \\ Patee Gesuwan, Leandro C Hermida, Bodour Salhia, Stephen Capone, Giridharan Ramsingh, Christian Michel Zwaan, Sanne Noort, \\ Stephen R Piccolo, E Anders Kolb, Alan S Gamis, Malcolm A Smith, Daniela S Gerhard \& Soheil Meshinchi \\ Nat. Med.; 10.1038/nm.4439; corrected online 1 January 2018
}

In the version of this article initially published online, Figure 1a has two black boxes in the key that are labeled as 'Unknown'; these boxes should be white, matching the segments in the donut charts shown below the key. The error has been corrected in the print, PDF and HTML versions of this article.

\section{Erratum: Sorafenib promotes graft-versus-leukemia activity in mice and humans through IL-15 production in FLT3-ITD-mutant leukemia cells}

\begin{abstract}
Nimitha R Mathew, Francis Baumgartner, Lukas Braun, David O'Sullivan, Simone Thomas, Miguel Waterhouse, Tony A Müller, Kathrin Hanke, Sanaz Taromi, Petya Apostolova, Anna L Illert, Wolfgang Melchinger, Sandra Duquesne, Annette Schmitt-Graeff, Lena Osswald, Kai-Li Yan, Arnim Weber, Sonia Tugues, Sabine Spath, Dietmar Pfeifer, Marie Follo, Rainer Claus, Michael Lübbert, Christoph Rummelt, Hartmut Bertz, Ralph Wäsch, Johanna Haag, Andrea Schmidts, Michael Schultheiss, Dominik Bettinger, Robert Thimme, Evelyn Ullrich, Yakup Tanriver, Giang Lam Vuong, Renate Arnold, Philipp Hemmati, Dominik Wolf, Markus Ditschkowski, Cordula Jilg, Konrad Wilhelm, Christian Leiber, Sabine Gerull, Jörg Halter, Claudia Lengerke, Thomas Pabst, Thomas Schroeder, Guido Kobbe, Wolf Rösler, Soroush Doostkam, Stephan Meckel, Kathleen Stabla, Stephan K Metzelder, Sebastian Halbach, Tilman Brummer, Zehan Hu, Joern Dengjel, Björn Hackanson, Christoph Schmid, Udo Holtick, Christof Scheid, Alexandros Spyridonidis, Friedrich Stölzel, Rainer Ordemann, Lutz P Müller, Flore Sicre-de-Fontbrune, Gabriele Ihorst, Jürgen Kuball, Jan E Ehlert, Daniel Feger, Eva-Maria Wagner, Jean-Yves Cahn, Jacqueline Schnell, Florian Kuchenbauer, Donald Bunjes, Ronjon Chakraverty, Simon Richardson, Saar Gill, Nicolaus Kröger, Francis Ayuk, Luca Vago, Fabio Ciceri, Antonia M Müller, Takeshi Kondo, Takanori Teshima, Susan Klaeger, Bernhard Kuster, Dennis (Dong Hwan) Kim, Daniel Weisdorf, Walter van der Velden, Daniela Dörfel, Wolfgang Bethge, Inken Hilgendorf, Andreas Hochhaus, Geoffroy Andrieux, Melanie Börries, Hauke Busch, John Magenau, Pavan Reddy, Myriam Labopin, Joseph H Antin, Andrea S Henden, Geoffrey R Hill, Glen A Kennedy, Merav Bar, Anita Sarma, Donal McLornan, Ghulam Mufti, Betul Oran, Katayoun Rezvani, Omid Shah, Robert S Negrin, Arnon Nagler, Marco Prinz, Andreas Burchert, Andreas Neubauer, Dietrich Beelen, Andreas Mackensen, Nikolas von Bubnoff, Wolfgang Herr, Burkhard Becher, Gerard Socié, Michael A Caligiuri, Eliana Ruggiero, Chiara Bonini, Georg Häcker, Justus Duyster, Jürgen Finke, Erika Pearce, Bruce R Blazar \& Robert Zeiser Nat. Med. 24, 282-291 (2018); published online 12 February 2018; corrected after print 6 March 2018
\end{abstract}

In the version of this article initially published, Omid Shah's name was misspelled as Omid Sha. The error has been corrected in the PDF and HTML versions of this article. 